\title{
MALBAC-based chromosomal imbalance analysis: a novel technique enabling effective non-invasive diagnosis and monitoring of bladder cancer
}

Hao Liu ${ }^{1,2}$, Wang He $e^{1,2}$, Bo Wang ${ }^{1,2}$, Kewei $X u^{1,2}$, Jinli Han ${ }^{1,2}$, Junjiong Zheng ${ }^{1,2}$, Jun Ren ${ }^{3}$, Lin Shao ${ }^{3}$, Shiping Bo ${ }^{3}$, Sijia Lu ${ }^{3 *}$, Tianxin $\operatorname{Lin}^{1,2^{*}}$ and Jian Huang ${ }^{1,2^{*}}$

\begin{abstract}
Background: The gold standard for bladder cancer detection is cystoscopy, which is an invasive procedure that causes discomfort in patients. The currently available non-invasive approaches either show limited sensitivity in low-grade tumours or possess unsatisfying specificity. The aim of the present study is to develop a new non-invasive strategy based on chromosomal imbalance levels to detect bladder cancer effectively.

Methods: We enrolled 74 patients diagnosed with bladder cancer (BC), 51 healthy participants and 27 patients who were diagnosed with non-malignant urinary disease (UD). The Chromosomal Imbalance Analysis (CIA) was conducted in the tumours and urine of participants via the multiple annealing and looping-based amplification cycles-next-generation sequencing (MALBAC-NGS) strategy. The threshold of the CIA was determined with the receiver operating characteristic (ROC) curve. The comparison of the CIA with voided urine cytology was also performed in a subgroup of 55 BC patients. The consistency and discrepancy of the different assays were studied with the Kappa analysis and the McNemar test, respectively. The performance of the urinary CIA was also validated in an additional group of 120 BC patients, 15 UD and 45 healthy participants.

Results: Good concordance (87.0\%) in the assessments of patient tumour tissues and urine was observed. The urinebased evaluation also demonstrated a good performance (accuracy $=89.0 \%$, sensitivity $=83.1 \%$, specificity $=94.5 \%$, NPV $=85.4 \%$ and PPV $=93.7 \%$; AUC $=0.917,95 \% \mathrm{Cl}=0.868-0.966, P<0.001)$ in the training group, particularly in the patients with CIA-positive tumours (accuracy $=92.7 \%$, sensitivity $=89.8 \%$ ). The sensitivity and specificity in the validation group were 89.2 and $90.0 \%$, respectively. Even in $\mathrm{Ta} / \mathrm{T} 1$ and low-grade tumour patients, the sensitivity was $85-90 \%$. The CIA also exhibited a significantly improved sensitivity compared to voided urine cytology.

Conclusions: This is the first study employing the concept of whole genome imbalance combined with the MALBAC technique to detect bladder cancer in urine. MALBAC-CIA yielded significant diagnostic power, even in early-stage/lowgrade tumour patients, and it may be used as a non-invasive approach for diagnosis and recurrence surveillance in bladder cancer prior to the use of cystoscopy, which would largely reduce the burden on patients.
\end{abstract}

Keywords: Bladder Cancer, CNV, MALBAC, NGS, Chromosomal imbalance analysis

\footnotetext{
* Correspondence: lusijia@yikongenomics.com; tianxinl@sina.com;

Urolhj@sina.com

${ }^{3}$ Department of Clinical Research, Yikon Genomics, 1698 Wangyuan Road

Building \#26, Fengxian District, Shanghai 201400, China

${ }^{1}$ Department of Urology, Sun Yat-Sen Memorial Hospital, Sun Yat-Sen

University, 107 Yanjiangxi Road, Guangzhou, China

Full list of author information is available at the end of the article
}

(c) The Author(s). 2018 Open Access This article is distributed under the terms of the Creative Commons Attribution 4.0 International License (http://creativecommons.org/licenses/by/4.0/), which permits unrestricted use, distribution, and

reproduction in any medium, provided you give appropriate credit to the original author(s) and the source, provide a link to the Creative Commons license, and indicate if changes were made. The Creative Commons Public Domain Dedication waiver (http://creativecommons.org/publicdomain/zero/1.0/) applies to the data made available in this article, unless otherwise stated. 


\section{Background}

Bladder cancer (BC) is one of the most common cancers in the world. Approximately $90 \%$ of patients with bladder cancer present with urothelial carcinoma, which has a high recurrence rate [1-3]. However, the diagnosis and the follow-up monitoring of $\mathrm{BC}$ has remained a challenge due to the lack of disease-specific symptoms [4]. Cystoscopy, although generally accepted as the gold standard for $\mathrm{BC}$ detection and surveillance, is an invasive procedure.

Voided urine cytology is a standard non-invasive approach adjunct to cystoscopy. The technique is highly specific $(85-100 \%)$, but the sensitivity is tumour-grade dependent. Although good sensitivity was demonstrated for detecting high-grade urothelial cancer (80-90\%, [1]), the technique is poor in terms of the detection of low-grade tumours, ranging from only 4-31\% detection rates [4].

A profound number of new urinary biomarkers have been developed by laboratory and clinical investigations, many of which have also been approved by the FDA, such as NMP22, UroVysion ${ }^{\circ}$ (fluorescence in situ hybridization, FISH) and BTAstat. Although many of these tests exhibit better sensitivity than urine cytology (up to 70-80\%), they come with the price of lower specificity (median 70-85\%) compared to cytology and therefore need to be further improved for wider application [4-7]. Therefore, a non-invasive, convenient and affordable urine-based test with high sensitivity and specificity is urgently in demand for $\mathrm{BC}$ diagnosis and monitoring.

Chromosomal instability is a common feature of tumour cells and has been reported to correlate with the development of bladder cancer [8]. Chromosomal instability might cause genomic abnormalities, such as alterations in chromosomal numbers and loss and/or gain of DNA in certain chromosomal segments $[9,10]$. It has been recently reported that chromosome instability has the potential to function as a prognostic predictor in non-small-cell lung carcinoma [11]. In the present study, we developed a next-generation sequencing (NGS)-based evaluation approach, the chromosomal imbalance analysis (CIA), in combination with a previously reported whole genome amplification (WGA) technique of multiple annealing and looping-based amplification cycles (MALBAC) [12] to assess the chromosomal aberration level of cells in urine, and demonstrated the application of the MALBAC-CIA for BC detection.

\section{Methods}

\section{Patient information}

The study design is demonstrated in Additional file 1: Figure S1. In the training group, a total of 74 patients diagnosed with BC in Sun Yat-sen Memorial Hospital, Sun Yat-sen University were recruited from 2015 to 2016 . We also enrolled 51 healthy participants and 23 patients who were diagnosed with non-malignant urinary disease (UD) as controls. For validation, 120 BC patients, 15 UD patients and 45 healthy participants from 2017 to 2018 were enrolled. Written informed consent was obtained from all participants, and the study was approved by the Medical Ethics Committee of Sun Yat-sen Memorial Hospital, Sun Yat-sen University. Tumour tissues were collected from $57 \mathrm{BC}$ patients in the training group at the time of transurethral resection of bladder tumour (TURBT) or cystectomy and were stored at $-80{ }^{\circ} \mathrm{C}$. The urine samples were collected from the second voided urine in the morning from all the participants, as shown in Additional file 1: Figure $\mathrm{S} 1$, and were stored at $-80{ }^{\circ} \mathrm{C}$.

\section{Next-generation sequencing}

DNA was extracted from the tumour tissues according to the manufacturer's instructions (Qiagen, Germany). First, $50 \mathrm{ml}$ of urine was centrifuged at $1600 \mathrm{~g}$ for $10 \mathrm{~min}$ to obtain cell pellets. The pellets were then washed and suspended in phosphate-buffered saline (PBS). Both the extracted DNA and the cell pellets were then subjected to NGS library preparation using the MALBAC-LIB kit (Yikon Genomics, China KT100800124) following the manufacturer's instructions. The sequencing was performed on an Illumina HiSeq 2500 sequencer. Approximately $5 \mathrm{M}$ sequencing reads were obtained from each sample.

\section{Chromosomal copy number variation (CNV) and CIA calculation}

The adaptors and low-quality bases were removed from the raw data. High quality reads were mapped to the hg19 reference genome using BWA (version 0.7.12-r1039) with default parameters. Unique mapped reads were extracted from the alignment reads (.bam file). The whole reference genome was divided into non-overlapped observation windows (bins) with a size of $1000 \mathrm{~Kb}$.

The relative copy number (xi) of each bin was calculated accordingly [12]. In brief, the read number and GC content were calculated in each bin. The bin read count was normalized based on the GC content and on a reference dataset to represent the relative copy number (xi). The $\mathrm{R}$ programming language was used to graph the $\mathrm{xi}$ of each bin to visualize copy number variations. Then, the $\mathrm{Z}$ value of each bin was calculated according to the formula:

$$
\mathrm{Z}_{\mathrm{i}}=\sqrt{\left|\log _{2}\left(\frac{x_{i}}{2}\right)\right|}
$$

The CIA score was calculated according to the formula: 


$$
\text { CIA score }=\sum_{\mathrm{i}=\mathrm{m}_{\mathrm{b}}}^{\mathrm{P}_{\mathrm{b}}}\left|\mathrm{Z}_{\mathrm{i}}\right|
$$

where $\mathrm{mb}$ and $\mathrm{pb}$ are the bins ranked $\mathrm{m} \%$ and $\mathrm{p} \%$, respectively, according to the $\mathrm{Z}$ value $(\mathrm{m}=95, p=99)$.

\section{Voided urine cytology}

Specimens from 57 of the 74 BC patients from the training group were prepared for urine cytology by the centrifuge and Cytospin methods. The slides were then stained and subjected to analysis by cytopathologists following the standard protocol. The evaluation of the test was recorded as negative, suspicious positive or positive.

\section{Statistical analysis}

A receiver operating characteristic (ROC) curve was generated for the urine samples from $\mathrm{BC}$ patients and controls from the training group to determine the threshold of the CIA score and to evaluate its performance (Additional file 1: Figure S1). Fisher's exact test was used to investigate the CIA score distribution in different T-stages and grades. The Kappa test was used to assess the consistency of CIA in the tissue and urine. The discrepancy from the various approaches was analysed by the McNemar test. All the analyses were performed with SPSS 22.0 (IBM, Chicago, IL, USA).

\section{Results}

The characteristics of the participants

A total of 74 patients diagnosed with $\mathrm{BC}$ were recruited in the training group, with a median age of 62 . Of those, 57 patients provided tumour tissues, and all (74) provided a urine sample (Additional file 1: Figure S1). All 57 tissue and 71/74 urine samples generated qualified NGS data for the CIA analysis, among which 54 patients had tissue samples paired with urine samples. Fifty-one healthy participants and 23 UD patients provided urine samples for the subsequent CIA analysis as controls (Additional file 1: Figure S1). For the validation set, urine CIAs of an additional $120 \mathrm{BC}$ patients, $15 \mathrm{UD}$ patients and 45 healthy participants were calculated. The demographic and clinical information for all the participants, including the TNM stage and histologic grade, are summarized in Table 1, and the details are listed in Additional file 2: Tables S1-3.

\section{The cut-off determination and distribution of $\mathrm{CIA}$}

The cut-off of CIA was determined by performing the ROC curve on all 145 urine samples from the training group $(71 \mathrm{BC}, 23 \mathrm{UD}$ and 51 healthy participants, Additional file 1: Figure S1). A cut-off of 24 was ultimately determined (Fig. 1), which yielded an accuracy of $89.0 \%$ and an area under the curve (AUC) of 0.917 (95\%CI $=0.868-0.966, P<0.001)$. The sensitivity and the

Table 1 The demographic and clinical characteristics of the participants

\begin{tabular}{|c|c|c|c|c|c|c|}
\hline & \multicolumn{3}{|c|}{ Training Group } & \multicolumn{3}{|c|}{ Validation Group } \\
\hline & $\mathrm{BC}$ & UD & $\mathrm{NC}$ & $B C$ & UD & NC \\
\hline Total No. & 74 & 23 & 51 & 120 & 15 & 45 \\
\hline \multicolumn{7}{|l|}{ No. gender (\%) } \\
\hline Female & $7(9.5)$ & $8(34.8)$ & $15(29.4)$ & $21(17.5)$ & $5(33.3)$ & $9(20)$ \\
\hline Male & $67(90.5)$ & $15(65.2)$ & $36(70.6)$ & $99(82.5)$ & $10(66.7)$ & $36(80)$ \\
\hline Median age (range) & $62(28-90)$ & $56(18-72)$ & $41(23-69)$ & $62(33-93)$ & $54(22-73)$ & $40(21-66)$ \\
\hline Primary (\%) & $61(82.4)$ & - & - & $106(88.3)$ & - & - \\
\hline Recurrent (\%) & $13(17.6)$ & - & - & 14(11.7) & - & - \\
\hline \multicolumn{7}{|l|}{ No. tumour stage (\%) } \\
\hline Tis & $1(1.4)$ & - & - & $2(1.7)$ & - & - \\
\hline $\mathrm{Ta}$ & $23(31.1)$ & & & $34(28.3)$ & & \\
\hline $\mathrm{T} 1$ & $24(32.4)$ & & & $61(50.8)$ & & \\
\hline $\mathrm{T} 2$ & $16(21.6)$ & & & $13(10.8)$ & & \\
\hline T3 & $5(6.8)$ & & & $10(8.3)$ & & \\
\hline NA & $5(6.8)$ & & & $0(0.0)$ & & \\
\hline \multicolumn{7}{|l|}{ No. tumour grade (\%) } \\
\hline PUNLMP & $5(6.8)$ & - & - & $0(0.0)$ & - & - \\
\hline LG & $9(12.2)$ & & & $33(27.5)$ & & \\
\hline$H G$ & $59(79.7)$ & & & $83(69.2)$ & & \\
\hline NA & $1(1.4)$ & & & $4(3.3)$ & & \\
\hline
\end{tabular}

$B C$ bladder cancer patients, UD non-malignant urinary disease patients, $N C$ healthy participants 


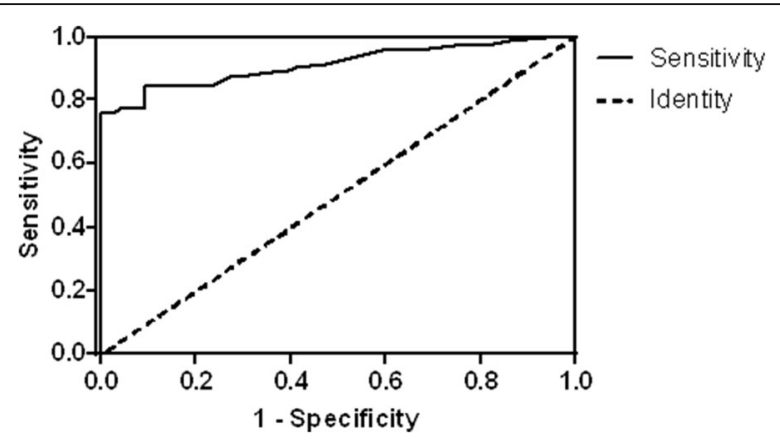

Fig. 1 ROC curve analysis for urine CIA scores. To determine the best cut-off value that discriminated between malignant BC patients and control groups, urine CIA scores from 71 BC, 23 UD and 51 healthy participants were included. The cut-off was defined as 24 [Accuracy = $89.0 \%$, sensitivity $=83.1 \%$, specificity $=94.5 \%, \mathrm{NPV}=85.4 \%$ and PPV $=$ 93.7\%]. Area under the curve (AUC) $=0.917,95 \% \mathrm{Cl}$ $=0.868-0.966, P<0.001$

specificity were 83.1 and $94.5 \%$, respectively. The negative predictive value (NPV) and the positive predictive value (PPV) were 85.4 and $93.7 \%$, respectively.

As illustrated in Fig. 2a, the BC patients were distinguished from the healthy participants and the UD patients by the urine CIA score. In particular, 83.1\% (59/71) of the $\mathrm{BC}$ patients had CIA scores above the cut-off of 24 . On the other hand, the scores of the healthy control and UD patients were mainly clustered under 24 , with only $3.9 \%$ $(2 / 51)$ of the former group and $8.7 \%(2 / 23)$ of the latter group being greater than the cut-off. In the 57 tumour tissues, $52(91.2 \%)$ had CIA scores above the cut-off of 24 (Fig. 2a), and 3 of them failed to generate paired urine outcomes (Additional file 2: Table S1). In the remaining 49 tumour CIA + patients, concordant positive urine CIA scores were observed in 44 (89.8\%).

The distribution of tissue and urine CIA scores in different TNM stages and grades are displayed in Fig. 2b, c. No significant differences were observed for CIA-positive and negative proportions among the various stages or grades $(P>0.05)$.

\section{The concordance of assessment between urine and tumour tissues via $\mathrm{CIA}$}

The chromosomal CNV profiles were investigated in the urine and paired tumour tissues of $\mathrm{BC}$ patients. The results showed similar CNV patterns in the urine and tissue of the same patient but diverse profiles among different individuals (Fig. 3). Then, the CIA scores were compared between the two types of samples. In 54 patients whose tumour tissues and paired urine were provided, 3 (5.6\%) were CIA-negative and 44 (81.5\%) were identified as CIA-positive in both urine and tissue samples (Table 2). Seven patients $(13.0 \%)$ had inconsistent outcomes between urine and tissue samples. The concordance rate was calculated as $87.0 \%$ (Kappa $=$
0.392, $P=0.003$ ), and no significant discrepancy in the evaluation was detected between the two types of samples, according to the McNemar Test $(P=0.453)$.

\section{Screening of BC patients by urine CIA score}

The performance of the CIA scores as a marker to distinguish $\mathrm{BC}$ patients from healthy participants and UD patients was assessed and is shown in Table 3. The overall sensitivity and accuracy were 83.1 and $89.0 \%$, respectively. Interestingly, when we limited the $\mathrm{BC}$ patients into the subgroup with CIA-positive tissues, the sensitivity was improved to $89.8 \%$. The accuracy also increased to $92.7 \%$.

Furthermore, we investigated the performance of CIA in various subgroups of $\mathrm{BC}$ patients with different TNM stages and histology grades (Table 3). The performance in muscle-invasive BC (T2 and T3) showed higher sensitivity than in non-muscle-invasive BC (Ta and T1). Notably, in the subset of patients with CIA-positive tissues, the sensitivity approached $90 \%$ for $\mathrm{Ta}+\mathrm{T} 1$ and was $100 \%$ for $\mathrm{T} 2+$ T3 in our cohort. Regarding the histological grade, the CIA resulted in higher sensitivity in histologically low-grade (LG) than histologically high-grade (HG) patients $(88.9 \%$ vs. $83.9 \%)$. However, the trend was the opposite in the subgroup of tissue CIA + patients (HG vs. LG: $92.5 \%$ vs. $85.7 \%)$. This discrepancy might be attributed to the limited number of LG patients enrolled in the study. In the 5 papillary urothelial neoplasms of low malignant potential (PUNLMP) patients, the sensitivity of CIA was not satisfying (60\%).

\section{The comparison of the $\mathrm{CIA}$ with voided urine cytology} Voided urine cytology was also conducted in $57 \mathrm{BC}$ patients from the training group. Among these patients, the urine CIA analysis was successfully achieved in 55 patients, and the results were compared (Table 4). Only 29 (52.7\%) of the BC patients were identified as positive by cytology, whereas $44(80 \%)$ urine samples were detectable by CIA. This improvement was significant $(p=0.004)$.

\section{The validation of $\mathrm{CIA}$ score in detecting $\mathrm{BC}$ patients}

To validate the established CIA score in discriminating $\mathrm{BC}$ patients, a validation cohort of $120 \mathrm{BC}$ patients and 60 control participants was recruited. The CIA resulted in a sensitivity of $89.2 \%$ and a specificity of $90.0 \%$ in the validation set (Table 5). Particularly, the sensitivity was $83.3 \%$ in Tis/Ta patients, and it increased to 88.5 and $100 \%$ in $\mathrm{T} 1$ and $\mathrm{T} 2 / \mathrm{T} 3$ patients, respectively. In terms of tumour grade, HG patients showed a higher sensitivity compared to LG patients (90.4\% vs. $84.8 \%)$.

\section{Discussion}

Chromosomal aberration is a common occurrence in tumours. In addition to aneuploidy, alterations in chromosomal architecture, focal amplifications and deletions are 


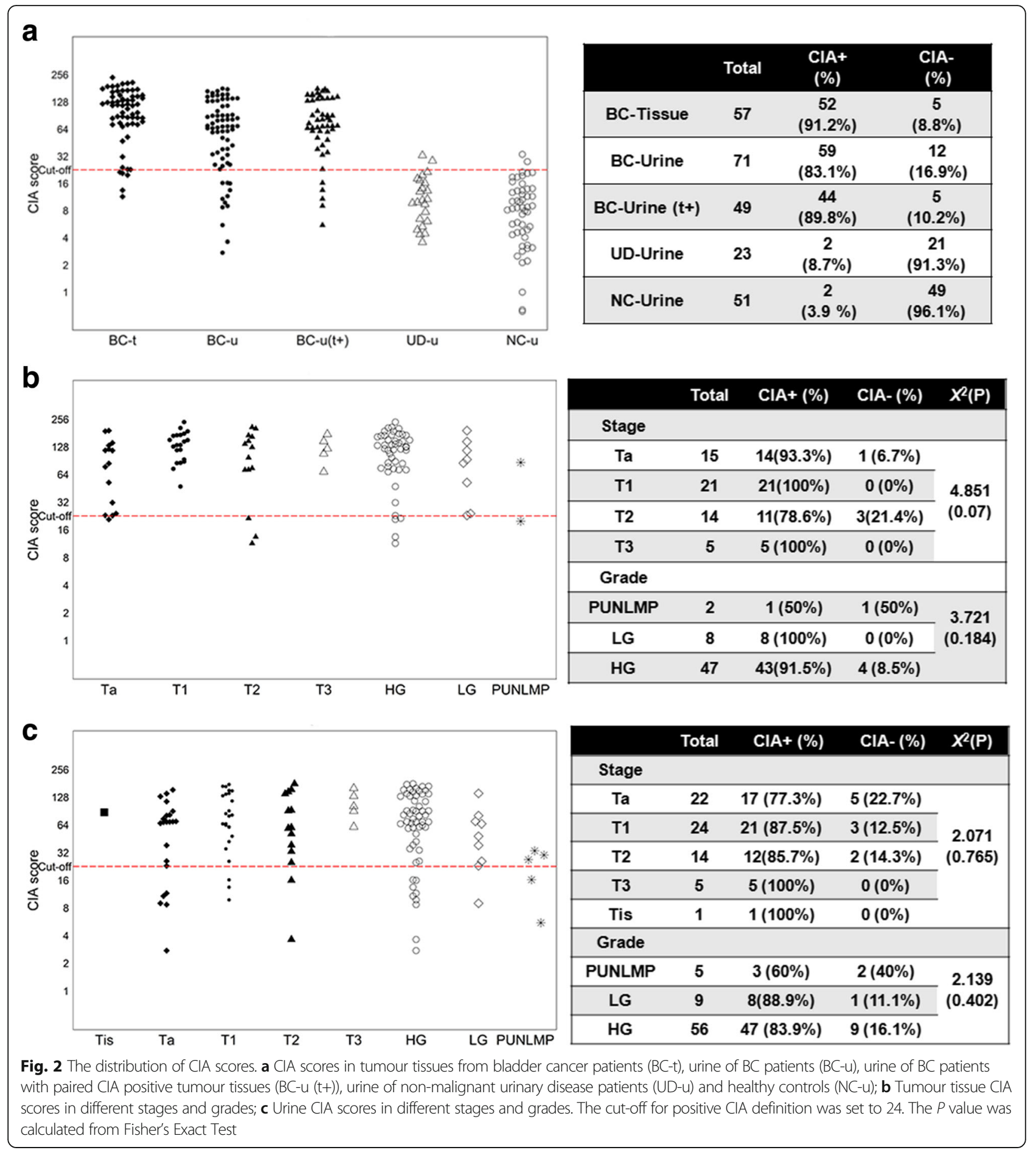

observed in cancer genomes. As a driving factor, the chromosomal abnormality manifests at the earliest stages of tumourigenesis and accumulates throughout subsequent tumour development [9, 13-15]. The urinary FISH test (UroVysion ${ }^{\circ}$ ), which probes alterations in chromosomes $3,7,17$ and 9p21, is one of the commercially available urinary biomarkers used to detect $\mathrm{BC}$. The sensitivity and specificity have been reported in systematic reviews and meta-analyses to exceed $70 \%$ and $\sim 80 \%$, respectively, but with a broad range among different studies [16-18]. The methylation patterns of a number of candidate genes have also been explored as potential biomarkers [16, 19]. More recently, a combination of methylation status of TWIST, ONECUT2, and OTX1 with mutational analyses 


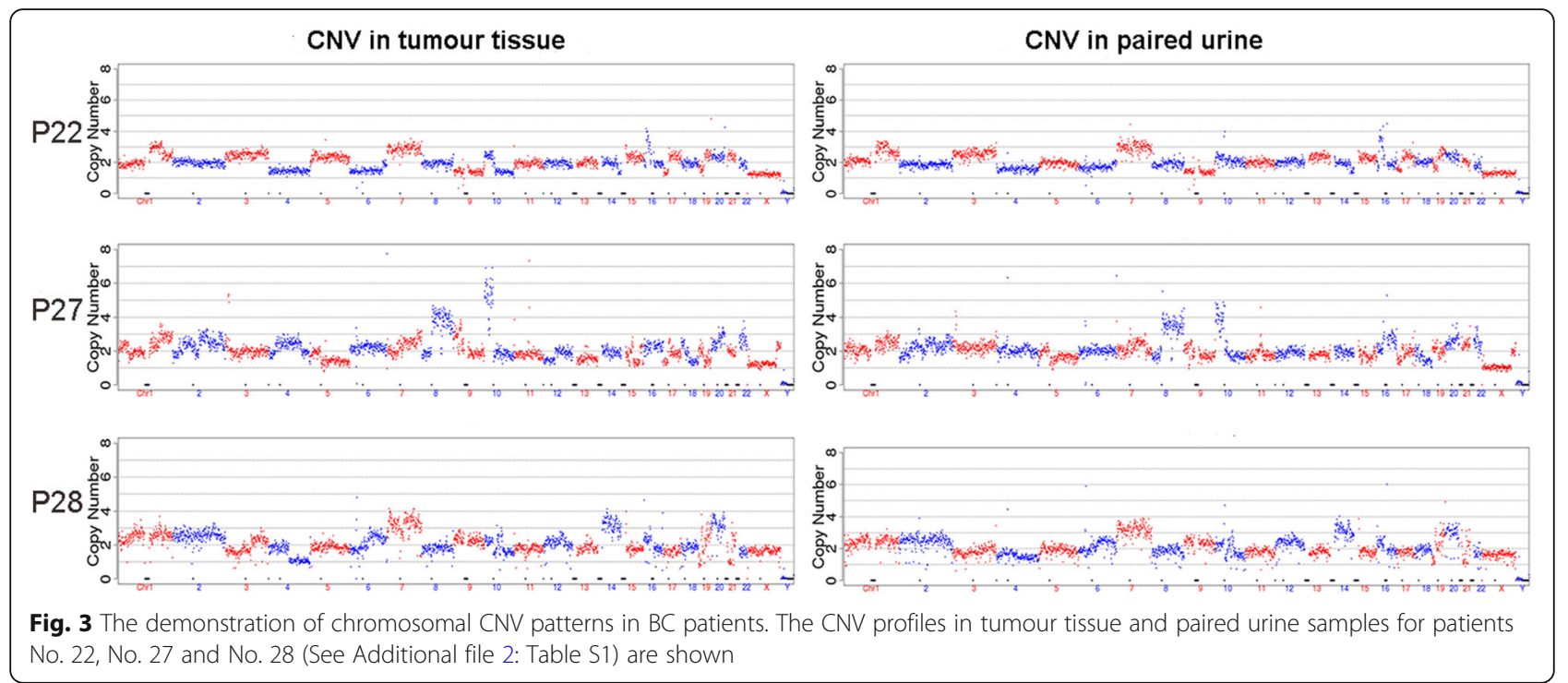

of FGFR3, TERT, and HRAS has been reported to detect bladder cancer with a sensitivity of $97 \%$ and a specificity of $83 \%$ [20, 21]. However, due to the diversity of the tumourigenesis driver mutations and the randomness of the somatic passenger mutations [22], tremendous genetic heterogeneity is spatially and temporally observed in tumour cells and is expected in different individuals, as is the case in bladder cancer [23] (Fig. 3). Conceivably, using the manifestation of genomic abnormality/imbalance that comprehensively assesses the variation across the whole genome as an indicator of bladder cancer detection might show superior sensitivity. Previously, different evaluation scores based on whole-genome sequencing were reported in prostate, colorectal and breast cancers for diagnosis and prognosis among limited numbers of patients/controls $[9,24-26]$. It is known that the number of exfoliated tumour cells varies in BC patients. This amount may be associated with the size and grade of the tumour. Therefore, an approach capable of detecting a small number of exfoliated tumour cells is in demand for urine-based diagnosis. As a commonly used WGA technique for single cell genome studies, MALBAC facilitates the analysis of trace

Table 2 Concordance of CIA evaluation between tumour tissues and urine samples. $(N=54)$

\begin{tabular}{lclr}
\hline ClA in urine & \multicolumn{2}{c}{ CIA in tissue } & Total (\%) \\
\cline { 2 - 3 } & Negative (\%) & Positive (\%) & \\
\hline Negative (\%) & $3(5.6 \%)$ & $5(9.3 \%)$ & $8(14.8 \%)$ \\
Positive (\%) & $2(3.7 \%)$ & $44(81.5 \%)$ & $46(85.2 \%)$ \\
Total (\%) & $5(9.3 \%)$ & $49(90.7 \%)$ & $54(100 \%)$ \\
Concordance (\%) & $87.0 \%$ & & \\
Kappa & $0.392, P=0.003$ & & \\
McNemar Test & $P=0.453$ & & \\
\hline
\end{tabular}

amounts of starting materials and does not require additional DNA extraction [12, 27]. MALBAC possesses the advantages of convenience and rapidness compared to a routine library construction process for NGS.

In the present study, we developed a novel strategy based on NGS that incorporates MALBAC and a new chromosomal imbalance evaluation approach, CIA, to assess the aberrant level of the chromosomal genome, and we demonstrated its application in detecting $\mathrm{BC}$ for the first time. Approximately $92 \%$ of the $\mathrm{BC}$ patients were identified as positive in tumour tissues according to the CIA, regardless of the TNM stage and histological grade, indicating its potentially wider utility for diagnosis. Moreover, as demonstrated in Fig. 3, the CNV profile of urine shows characteristics similar to tissues derived from the same patient, suggesting that urine cell pellets are representative of tumours for $\mathrm{CNV}$ assessment. The urine CIA also exhibits concordance with tissue CIA, indicating its potential as a non-invasive diagnostic strategy (Table 2). Notably, the performance of urine CIA was superior in the subgroup of patients carrying CIA-positive tumours than in all the patients. Tissue CIA might serve as a prior test to select patients possessing positive CIA scores in primary tumours for the subsequent recurrence surveillance by non-invasive urine CIA. Nonetheless, the heterogeneity in primary and recurrent tumours should also be taken into account, and the feasibility of this method needs to be investigated and validated by prospective studies.

The performance of this technique was also compared with other non-invasive methods. Voided urine cytology is routinely used in the clinic with good specificity (>90\%); however, the sensitivity has been reported to be $30-50 \%$ [4]. CIA showed significantly improved sensitivity in detecting BC patients in this study $(80-90 \%$ vs. $52.7 \%)$, 
Table 3 The performance of the urine CIA score in training group to distinguish BC patients in different TNM stages and histology grades

\begin{tabular}{|c|c|c|c|c|}
\hline $\mathrm{BC}$ patients & No. of (Case/Control) & Sensitivity & Specificity & Accuracy \\
\hline All patients & $71 / 74$ & $83.1 \%$ & $94.5 \%$ & $89.0 \%$ \\
\hline $\mathrm{Tis}+\mathrm{Ta}+\mathrm{T} 1$ & $47 / 74$ & $83.0 \%$ & $94.5 \%$ & $90.1 \%$ \\
\hline $\mathrm{T} 2+\mathrm{T} 3$ & $19 / 74$ & $94.7 \%$ & $94.5 \%$ & $94.6 \%$ \\
\hline PUNLMP & $5 / 74$ & $60 \%$ & $94.5 \%$ & $92.4 \%$ \\
\hline LG & $9 / 74$ & $88.9 \%$ & $94.5 \%$ & $94.0 \%$ \\
\hline$H G$ & $55 / 74$ & $83.9 \%$ & $94.5 \%$ & $90.0 \%$ \\
\hline With CIA+ Tissues & $49 / 74$ & $89.8 \%$ & $94.5 \%$ & $92.7 \%$ \\
\hline $\mathrm{Ta}+\mathrm{T} 1$ & $34 / 74$ & $88.2 \%$ & $94.5 \%$ & $92.6 \%$ \\
\hline $\mathrm{T} 2+\mathrm{T} 3$ & $14 / 74$ & $100 \%$ & $94.5 \%$ & $95.5 \%$ \\
\hline PUNLMP & $1 / 74$ & $0 \%$ & $94.5 \%$ & $93.3 \%$ \\
\hline LG & $8 / 74$ & $87.5 \%$ & $94.5 \%$ & $93.9 \%$ \\
\hline$H G$ & $40 / 74$ & $92.5 \%$ & $94.5 \%$ & $93.9 \%$ \\
\hline
\end{tabular}

which is also superior to the reported cytology sensitivity in the literature. The FISH (UroVysion ${ }^{\circ}$ ) probes alterations in chromosomes 3, 7, 17 and 9p21 and the sensitivity and specificity have been reported to be $\sim 50-80 \%$ and 70 $85 \%$, respectively $[4,17,18]$. CIA displayed a superior performance, with both the sensitivity and specificity being $90 \%$ in the training and validation groups. Other commercially available markers (such as NMP22, ImmunoCyt and BTA stat) also show unsatisfactory performance $[4,16]$.

One major limitation of the currently available urinary biomarkers is the poor sensitivity for early-stage and lower-grade tumours $[1,4,18]$. However, $30-80 \%$ of patients diagnosed with a low-grade $\mathrm{Ta} / \mathrm{T} 1$ primary tumour undergo recurrence within 5 years [1-3]. In addition, tumours generally are larger or in a more advanced stages at diagnosis than during surveillance. A non-invasive test with high sensitivity, particularly for early-stage and low-grade tumours, is important for the surveillance of patients by reducing the use of invasive tests such as cystoscopy and thereby improving the patient quality of life [1]. However, it has been reported that cells from men with low-grade BC accumulated fewer CNVs than those from cases with high-grade cancer [28]. Hurst et al. [29] also discovered that the more genomically unstable subtype of Ta bladder cancer was distinguished by loss of chromosome 9q, and the

Table 4 The comparison of the CIA results with voided urine cytology in BC patients

\begin{tabular}{llll}
\hline Urine CIA & \multicolumn{2}{l}{ Cytology } & Total (\%) \\
\cline { 2 - 3 } & Negative (\%) & Positive (\%) & \\
\hline Negative (\%) & $6(10.9 \%)$ & $5(9.1 \%)$ & $11(20.0 \%)$ \\
Positive (\%) & $20(36.4 \%)$ & $24(43.6 \%)$ & $44(80.0 \%)$ \\
Total (\%) & $26(47.3 \%)$ & $29(52.7 \%)$ & $55(100 \%)$ \\
McNemar Test & $P=0.004$ & & \\
\hline
\end{tabular}

The original cytology results included negative, suspicious positive and positive. The results of suspicious positive and positive were regarded as positive in this comparison other subtype contained no or few copy-number alterations. This outcome might explain the observation that the CIA showed a slightly lower sensitivity in early-stage and low-grade tumours (83-85\%) than that of more advanced stage and high-grade tumours (90-100\%). However, the observed sensitivity is better than the performance of other commercial markers in the same stage/grade, which have been reported to be less than $80 \%$ in most cases $[1,4$, 18]. The sensitivity of CIA was observed to be only $60 \%$ in the 5 PUNLMP patients, which might be explained by its low level of malignancy. However, a limited number of patients were included in the present study; therefore, a conclusive statement cannot be made without further validation in a larger set of patients.

The cost of this new technique is estimated to be $\$ 200-300$ per patient. Although this is relatively costly compared to the methylation and mutation combination assay reported recently (\$23) [21], with the rapid reductions in NGS cost, the CIA assay is expected to become cost effective in the near future. Due to the utility of MALBAC technology, the CIA assay does not require a large amount of DNA, which makes it a more suitable technique for urine-based testing. Compared to the DNA methylation assay, the minimum DNA input of which is approximately $50 \mathrm{ng}$ (the amount present in

Table 5 The validation of the urine CIA score to distinguish BC patients

\begin{tabular}{cllll}
\hline BC patients & No. of (Case/Control) & Sensitivity & Specificity & Accuracy \\
\hline All patients & $120 / 60$ & $89.2 \%$ & $90.0 \%$ & $89.4 \%$ \\
Tis + Ta & $36 / 60$ & $83.3 \%$ & $90.0 \%$ & $87.5 \%$ \\
T1 & $61 / 60$ & $88.5 \%$ & $90.0 \%$ & $89.3 \%$ \\
T2 + T3 & $23 / 60$ & $100.0 \%$ & $90.0 \%$ & $92.8 \%$ \\
LG & $33 / 60$ & $84.8 \%$ & $90.0 \%$ & $88.2 \%$ \\
HG & $83 / 60$ & $90.4 \%$ & $90.0 \%$ & $90.2 \%$ \\
\hline
\end{tabular}


8000 cells), the MALBAC assay is applicable to a single cell equivalent amount of DNA [12,27].

It has been reported that genetic mutations in certain genes, such as FGFR3, RB1, HRAS, TP53, TSC1, TERT and others, occur in urinary bladder tumours [30]. A proportion of urothelial tumours also harbour mutations that are potentially therapeutic targets, including the FGFR3, TSC1 and PIK3CA mutations [31-34]. It is quite possible that a combination of the reported CIA and other mutations might improve the detection rate of bladder cancers and might be informative for therapy selection.

\section{Conclusion}

Overall, we developed a new strategy based on the chromosomal imbalance/aberration level and demonstrated its application in $\mathrm{BC}$ detection for the first time. Good concordance (87.0\%) in the assessments obtained from patient tumours and urine was observed. The urine-based evaluation also demonstrated a good performance (accuracy $=89.0 \%$, sensitivity $=83.1 \%$, specificity $=94.5 \%, \mathrm{NPV}=85.4 \%$ and $\mathrm{PPV}=93.7 \% ; \mathrm{AUC}=0.917$, $95 \% \mathrm{CI}=0.868-0.966, P<0.001)$ in the training group, particularly in patients with CIA-positive tumours (accuracy $=92.7 \%$, sensitivity $=89.8 \%$ ). The performance was also validated in an additional group, with a sensitivity and specificity of $\sim 90 \%$. It is conceivable that the present approach might have the potential to be a non-invasive test for $\mathrm{BC}$ diagnosis and to be subsequent surveillance prior to cystoscopy use. We envision that prospective cohort studies, with larger samples incorporating both $\mathrm{BC}$ patients and a certain percentage of patients with related symptoms and/or signs, will be designed to further validate the feasibility of monitoring bladder cancer patients.

\section{Additional files}

Additional file 1: Figure S1. The illustration of the study design. The square ( $\square$ ) represents bladder cancer patients. The circle (o) and triangle ( $\boldsymbol{\Lambda}$ ) represent the healthy participants and patients diagnosed with non-malignant urinary diseases, respectively. The number refers to the number of participants (TIF $1991 \mathrm{~kb}$ ).

Additional file 2: The demographic and clinical characteristics of training group. Table S1. The demographic and clinical characteristics of bladder cancer (BC) patients of training group. The originally reported cytology classification included negative (-), suspicious (+-) and positive (+). Table S2. The demographic and clinical characteristics of non-malignant urinary disease (UD) patients of training group. Table S3. The demographic characteristics of healthy controls of training group (XLSX $27 \mathrm{~kb}$ ).

\section{Abbreviations}

CIA: Chromosomal Imbalance Analysis; BC: bladder cancer; NGS: next-generation sequencing; WGA: whole genome amplification; MALBAC: looping-based amplification cycle; UD: non-malignant urinary disease; CNV: Chromosomal copy number variation; ROC: receiver operating characteristic; TURBT: transurethral resection of bladder tumour; PBS: phosphate buffered saline; AUC: area under the curve; NPV: negative predictive value; PPV: positive predictive value; LG: low-grade; HG: high-grade; PUNLMP: papillary urothelial neoplasms of low malignant potential; FISH: fluorescence in situ hybridization

\section{Acknowledgements}

The authors would like to thank Dr. Xin Dong, Qinsi Liang and Shujie Ma for their assistance with the data analysis as well as Yunyun Niu and Ting Ma for their help with experimental support and data acquisition.

\section{Funding}

The study is supported by National Natural Science Foundation of China (Grant No. 81572514, U1301221, 81472384, 81402106, 81372729, 81272808, 81172431, 81772728, 81772719), National Key Research and Development Program of China (2016YFC1000702), National Natural Science Foundation of Guangdong (Grant No. 2016A030313321, 2015A030311011, 2015A030310122, S2013020012671), Science and Technology Program of Guangzhou (Grant No. 201604020156,

201604020177), "Three Big Constructions" funds of Sun Yat-sen University (for Jian Huang and Tianxin Lin), Specialized Research Fund for the Doctoral Program of Higher Education (for Tianxin Lin, 20130171110073), the Fundamental Research Funds for the Central Universities (for Jian Huang), Project Supported by Guangdong Province Higher Vocational Colleges \& Schools Pearl River Scholar Funded Scheme (for Tianxin Lin), Elite Young Scholars Program of Sun Yat-Sen Memorial Hospital (for Tianxin Lin, J201401), Sun Yat-sen Clinical Research Cultivating Program (for Hao Liu) and National Clinical Key Specialty Construction Project for Department of Urology and Department of Oncology, the Key Laboratory of Malignant Tumour Gene Regulation and Target Therapy of Guangdong Higher Education Institutes, Sun-Yat-Sen University (KLB09001) and the Key Laboratory of Malignant Tumour Molecular Mechanism and Translational Medicine of Guangzhou Bureau of Science and Information Technology (Grant [2013]163). All funding bodies have no role in the design of the study and collection, analysis, and interpretation of data and in writing the manuscript.

\section{Availability of data and materials}

The datasets used and/or analysed during the current study are available from the corresponding author on reasonable request.

\section{Authors' contributions}

$\mathrm{HL}, \mathrm{SL}, J \mathrm{H}$ and TL made substantial contributions to the conception and design and revised the manuscript. WH, BW, KX, JH and JZ conducted the experiments, contributed to data acquisition and drafted the manuscript. JR and SB analysed and interpreted the data. LS performed the statistical analysis and was a major contributor in writing the manuscript. All the authors read and approved the final manuscript.

\section{Ethics approval and consent to participate}

Written informed consent was obtained from all participants, and this study was approved by the Medical Ethics Committee of Sun Yat-sen Memorial Hospital, Sun Yat-sen University.

\section{Competing interests}

S. Lu is the co-founder of Yikon Genomics.

\section{Publisher's Note}

Springer Nature remains neutral with regard to jurisdictional claims in published maps and institutional affiliations.

\section{Author details}

'Department of Urology, Sun Yat-Sen Memorial Hospital, Sun Yat-Sen University, 107 Yanjiangxi Road, Guangzhou, China. ${ }^{2}$ Guangdong Provincial Key Laboratory of Malignant Tumour Epigenetics and Gene Regulation, Sun Yat-Sen Memorial Hospital, Sun Yat-Sen University, 107 Yanjiangxi Road, Guangzhou, China. ${ }^{3}$ Department of Clinical Research, Yikon Genomics, 1698 Wangyuan Road, Building \#26, Fengxian District, Shanghai 201400, China.

Received: 19 December 2017 Accepted: 31 May 2018

Published online: 15 June 2018

\section{References}

1. Lotan Y, O'Sullivan P, Raman JD, et al. Clinical comparison of noninvasive urine tests for ruling out recurrent urothelial carcinoma. Urol Oncol. 2017;35:531.

2. Bryan RT, Collins SI, Daykin MC, et al. Mechanisms of recurrence of ta/T1 bladder cancer. Ann R Coll Surg Engl. 2010;92(6):519-24. 
3. Goodison S, Rosser CJ, Urquidi V. Bladder cancer detection and monitoring: assessment of urine- and blood-based marker tests. Mol Diagn Ther. 2013; 17(2):71-84

4. Schmitz-Dräger BJ, Droller M, Lokeshwar VB, et al. Molecular markers for bladder cancer screening, early diagnosis, and surveillance: the WHO/ICUD consensus. Urol Int. 2015:94(1):1-24.

5. Eissa S, Matboli M, Shawky S, et al. Urine biomarkers of schistosomiais and its associated bladder cancer [J]. Expert Rev Anti-Infect Ther. 2015;13(8):985-93. https://doi.org/10.1586/14787210.2015.1051032.

6. Eissa S, Matboli M, Essawy NO, et al. Integrative functional geneticepigenetic approach for selecting genes as urine biomarkers for bladder cancer diagnosis [J]. Tumour Biol. 2015;36(12):9545-52. https://doi.org/10. 1007/s13277-015-3722-6.

7. Eissa S, Matboli M, Awad N, et al. Identification and validation of a novel autophagy gene expression signature for human bladder cancer patients [J]. Tumour Biol. 2017;39(4):1010428317698360. https://doi.org/10.1177/ 1010428317698360.

8. Florl AR, Schulz WA. Chromosomal instability in bladder cancer. Arch Toxicol. 2008;82(3):173-82.

9. Leary RJ, Sausen M, Kinde I, et al. Detection of chromosomal alterations in the circulation of cancer patients with whole-genome sequencing. Sci Transl Med. 2012;4(162):162ra154.

10. Bakhoum SF, Compton DA. Chromosomal instability and cancer: a complex relationship with therapeutic potential. J Clin Invest. 2012;122(4):1138-43.

11. Jamal-Hanjani M, Wilson GA, McGranahan N, et al. Tracking the evolution of non-small-cell lung Cancer [J]. N Engl J Med. 2017;376(22):2109-21. https:// doi.org/10.1056/NEJMoa1616288.

12. Zong C, Lu S, Chapman AR, Xie XS. Genome-wide detection of singlenucleotide and copy-number variations of a single human cell. Science. 2012;338(6114):1622-6.

13. Mitelman F, Johansson B, Mertens F. The impact of translocations and gene fusions on cancer causation. Nat Rev Cancer. 2007;7(4):233-45.

14. Campbell PJ, Stephens PJ, Pleasance ED, et al. Identification of somatically acquired rearrangements in cancer using genome-wide massively parallel paired-end sequencing. Nat Genet. 2008;40(6):722-9.

15. Stephens PJ, McBride DJ, Lin ML, et al. Complex landscapes of somatic rearrangement in human breast cancer genomes. Nature. 2009; 462(7276):1005-10.

16. Krabbe LM, Woldu SL, Shariat SF, et al. Improving diagnostic molecular tests to monitor urothelial carcinoma recurrence [J]. Expert Rev Mol Diagn. 2016; 16(11):1189-99. https://doi.org/10.1080/14737159.2016.1244006.

17. Gomella LG, Mann MJ, Cleary RC, et al. Fluorescence in situ hybridization (FISH) in the diagnosis of bladder and upper tract urothelial carcinoma: the largest single-institution experience to date. Can J Urol. 2017;24(1):8620-6.

18. Lavery HJ, Zaharieva B, McFaddin A, Heerema N. Pohar KS. A prospective comparison of UroVysion FISH and urine cytology in bladder cancer detection. BMC Cancer. 2017;17(1):247.

19. Eissa S, Swellam M, El-Khouly IM, et al. Aberrant methylation of RARbeta2 and APC genes in voided urine as molecular markers for early detection of bilharzial and nonbilharzial bladder cancer [J]. Cancer Epidemiol Biomark Prev. 2011:20(8):1657-64. https://doi.org/10.1158/1055-9965.EPI-11-0237.

20. van Kessel KE, Van Neste L, Lurkin I, et al. Evaluation of an epigenetic profile for the detection of bladder Cancer in patients with hematuria[J]. J Urol. 2016;195(3):601-7. https://doi.org/10.1016/j.juro.2015.08.085.

21. van Kessel KE, Beukers W, Lurkin I, et al. Validation of a DNA methylationmutation urine assay to select patients with hematuria for cystoscopy. J Urol. 2017:197(3 Pt 1):590-5.

22. Stratton MR, Campbell PJ, Futreal PA. The cancer genome. Nature. 2009; 458(7239):719-24

23. Chen C, Qi XJ, Cao YW, et al. Bladder tumor heterogeneity: the impact on clinical treatment. Urol Int. 2015;95(1):1-8.

24. Xia S, Kohli M, Du M, et al. Plasma genetic and genomic abnormalities predict treatment response and clinical outcome in advanced prostate cancer. Oncotarget. 2015;6(18):16411-21.

25. Xia $Y$, Huang CC, Dittmar $R$, et al. Copy number variations in urine cell free DNA as biomarkers in advanced prostate cancer. Oncotarget. 2016;7(24): 35818-31.

26. Heitzer E, Ulz P, Belic J, et al. Tumor-associated copy number changes in the circulation of patients with prostate cancer identified through wholegenome sequencing. Genome Med. 2013;5(4):30.
27. Lu S, Zong C, Fan W, et al. Probing meiotic recombination and aneuploidy of single sperm cells by whole-genome sequencing. Science. 2012; 338(6114):1627-30.

28. Bonberg N, Pesch B, Behrens T, et al. Chromosomal alterations in exfoliated urothelial cells from bladder cancer cases and healthy men: a prospective screening study [J]. BMC Cancer. 2014;14:854. https://doi.org/10.1186/14712407-14-854.

29. Hurst CD, Alder O, Platt FM, et al. Genomic subtypes of non-invasive bladder Cancer with distinct metabolic profile and female gender Bias in KDM6A mutation frequency [J]. Cancer Cell. 2017;32(5):701-715.e7. https:// doi.org/10.1016/j.ccell.2017.08.005.

30. Zhang X, Zhang Y. Bladder Cancer and genetic mutations. Cell Biochem Biophys. 2015;73(1):65-9.

31. Iyer G, Al-Ahmadie H, Schultz N, et al. Prevalence and co-occurrence of actionable genomic alterations in high-grade bladder cancer. J Clin Oncol. 2013;31(25):3133-40

32. Al-Ahmadie HA, lyer $\mathrm{G}$, Janakiraman $\mathrm{M}$, et al. Somatic mutation of fibroblast growth factor receptor-3 (FGFR3) defines a distinct morphological subtype of high-grade urothelial carcinoma. J Pathol. 2011;224(2):270-9

33. Iyer G, Hanrahan AJ, Milowsky MI, et al. Genome sequencing identifies a basis for everolimus sensitivity. Science. 2012;338(6104):221.

34. Ross JS, Wang K, Al-Rohil RN, et al. Advanced urothelial carcinoma: nextgeneration sequencing reveals diverse genomic alterations and targets of therapy. Mod Pathol. 2014;27(2):271-80.

\section{Ready to submit your research? Choose BMC and benefit from:}

- fast, convenient online submission

- thorough peer review by experienced researchers in your field

- rapid publication on acceptance

- support for research data, including large and complex data types

- gold Open Access which fosters wider collaboration and increased citations

- maximum visibility for your research: over $100 \mathrm{M}$ website views per year

At BMC, research is always in progress.

Learn more biomedcentral.com/submissions 